\title{
Artificial Neural Network (ANN) For Electronic Diagnosis in Telemedicine; Lagos University Teaching Hospital (LUTH)
}

\author{
P.B. Osofisan", H.A. Akanji ${ }^{2}$ \\ ${ }^{1}$ Faculty of Engineering, Department of Electronic and Computer Engineering. \\ Lagos State University, Lagos. Nigria \\ ${ }^{2}$ Faculty of Engineering, Department of Electrical and Electronic Engineering, \\ University of Lagos
}

Received: $17^{\text {th }}$ February 2018

Accepted: $10^{\text {th }}$ August 2018

Published: $20^{\text {th }}$ September 2018

https://doi.org/10.47545/etrj.2018.3.2.042

\begin{abstract}
Artificial Neural Network (AAN) has gained enormous popularity over the last one and half decades owing to its vast application in industry and research. The objective of this research work is to use ANN-based method to solve diagnosis problem in healthcare delivery, providing accurate electronic diagnosis and statistical analysis for telemedicine applications. The result showed that the software tool was able to identify $82 \%$ (45 out of 55 cases) as compared with medical test, 89\% (49 out of 55) as compared with the doctors' diagnosis.
\end{abstract}

Keywords: Telemedicine, Back propagation Neural Network, e-diagnosis, identification of symptoms and diseases.

\section{INTRODUCTION}

There have been attempts to develop software application system for e-physician to minimize the diagnosis error rate and to conduct effective diagnosis on the basis of real-time data of the patient [1]. Nowadays all the different areas of the healthcare market place (Physician, Patient and pharmacist) are challenged with providing effective diagnosis support system by providing electronic diagnosis and statistical analysis for telemedicine applications [13],[14], [15], [16]. A software application that produced an 'accurate' diagnosis (i.e. the same as a 'professional' would arrive at) at the end of some reasonable amount of processing was developed. In other words, a system was presented with a group of symptoms, and its job was to come up with a diagnosis, which was an identification of a disease or several diseases whose manifestations most closely match the condition of the patient. The availability of an e-Diagnosis at any time is a great advantage in telemedicine. Medicine has always benefited from the fore front of technology. Artificial Neural Network (ANN) using the feed forward multilayer back propagation has been successfully applied to this research work as it is extensively used in diagnostic systems. The e-Diagnosis system is used for detecting diseases such as ectopic pregnancy, menopause etc. The reason being that they are not affected by factors such as fatigue, working conditions and emotional state [2].The advantages of the e-Diagnosis system are obvious. Patients can be checked for diseases quickly and painlessly and thus detecting diseases at early stages. It may help in screening and can be used by experts to double-check their diagnosis. Late detection of diseases and deficiencies in patients' health state is also a problem in this part of the world. In addition, identification of diseases in emergency situation is always difficult in cases where there are no specialists to effectively carry out treatment. But applying Artificial Intelligence could optimize performance to eliminate the above problems as well as avoid repetition of medical test.

\section{LITERATURE REVIEW}

\section{e-Diagnosis procedure}

Most doctors use a combination of patient's case history and current symptoms to arrive at a medical diagnosis when a patient is ill. In order to recognize the combination of symptoms and medical history that points to a particular disease, the doctor's brain accesses the memory of previous patients, as well as information that has been learned from 
textbooks or other doctors [3]. A neural network has the tendency to imitate this type of decision-making process, and use a knowledge base of information, and a practical training set of data, to learn to diagnose diseases.

\section{Telemedicine}

Telemedicine is a rapidly developing application of clinical medicine where medical information is transferred through the phone or the Internet and sometimes other networks for the purpose of consulting, and sometimes remote medical procedures or examinations [1]. It is a priority tool in the delivery of health care as well as enhancing the knowledge of those working in the medical field. In other word, telemedicine is a combination of information and telecommunication technologies, which together provide medical services regardless of distance and medical personnel on site. The American Telemedicine Association defines it as the use of medical information exchanged from one site to another via electronic communication for the health and education of the patient or practitioner and for the purpose of improving patient care. It involves the electronic convergence of medical information for the purpose of diagnosis and treatments of patients [4]. It is also a useful tool in prevention or diagnosis of diseases, especially if they are dynamically lethal (deadly) such as cardiac disease. In isolated places, i.e. islands, mountainous areas or ships, where access to medical services is time consuming or infeasible, telemedicine could prove lifesaving [5].Telemedical research has tended to focus either on technological possibilities or evaluate criteria such as diagnostic accuracy.

\section{Benefits of Telemedicinee}

Telemedicine is most beneficial for populations living in remote communities and regions and is currently being applied in virtually all medical fields. Telemedicine is also useful as a communication tool between a general practitioner and a specialist available at a remote site [6]. Telemedicine is also an exciting new technique for healthcare delivery. The technique, acceptable to doctors and patients are enthusiastic about it; although it certainly changes the doctor-patient relationship, it does not do it irreversible damage. Telemedicine is not simply about technology and new equipment. It is a process [7], whose successful exploitation requires organizational changes in the structure of the relevant health care service. Given this, the experience suggests that in the right context telemedicine may be effective in improving access to healthcare, in raising the quality of service, and even reducing the costs.

\section{Current Telemedicine Application}

1) Cardiology 2) Dermatology 3) Dentistry 4) Endocrinology 5) Neurosurgery 6) Ophthalmology 7) Pediatrics 8) Urology 9) Surgery 10) Pulmonology 11) Internal Medicine 12) Emergency Medicine 13) Pediatry 14) Obstetrics and Gynaecology 15) Radiology

\section{Artificial Neural Network}

Neural Networks are a form of artificial intelligence that uses multiple artificial neurons, networked together to process information. In other words, it is similar to a computer software (and possibly hardware) that simulates a simple model of neural cells in animals and humans [8].The purpose of this simulation is to acquire the intelligent features of these cells. This type of network has the capability to learn from patterns, and extrapolate results from data that has been previously entered into the network's knowledge base. This ability makes neural network applications extremely valuable to the medical industry, particularly in the area of in-office, or medical diagnosis [3].Also with an ANN solution, you do not have to understand the solution at all. This is a major advantage of an ANN approach.

\section{Neural Network Architecture}

A neural network is made up of different layers; input layer, output layer, and hidden layer (for multi-layer networks).Two or more neurons are combined in a layer. We have the single layer network where each of the inputs is connected to each of the neurons, and it can solve problems that are linearly separable [2]. But in a case where the problem is non-linearly separable, additional layers will be added, forming a multi-layer network. In this work, a hidden layer will be required. A 3-layer network with $\mathrm{R}$ input is shown in Fig 1 


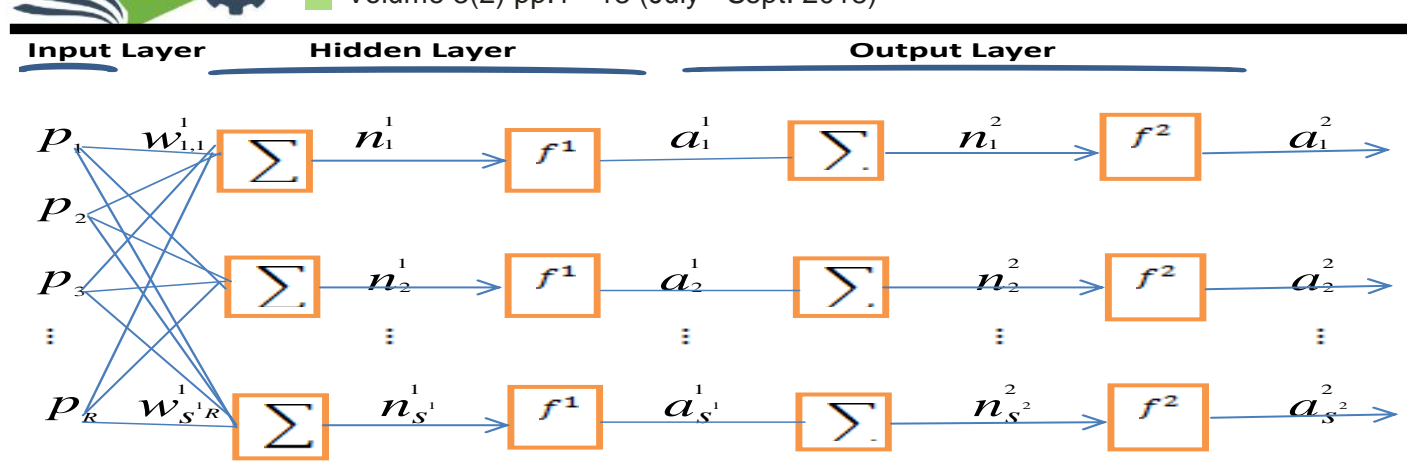

Fig. 1: A 3-Layer network with $\mathrm{R}$ input

Each layer has a net input vector $n$, output vector $\boldsymbol{a}$, and its own weight matrix $W$ (Martin, 2002)

Where $W=\left[\begin{array}{cccc}\mathcal{w}_{1,1} & w_{1,2} & \mathrm{~K} & \boldsymbol{w}_{1, R} \\ \mathcal{w}_{2,1} & \boldsymbol{w}_{2,2} & \mathrm{~K} & \boldsymbol{w}_{2, R} \\ \mathrm{M} & \mathrm{M} & & \mathrm{M} \\ \boldsymbol{w}_{S, 1} & \boldsymbol{w}_{S, 2} & \mathrm{~K} & \boldsymbol{w}_{S, R}\end{array}\right]$

$S=$ Number of neurons in a layer

A superscript is used to identify the different layer that is, superscript 0 stands for the input layer, superscript 1 stands for the hidden layer while superscript 2 stands for the output layer. The layer includes the weight matrix, the summers, the transfer function boxes, and the output vector. It is common for the number of inputs to a layer to be different from the number of neurons (i.e. $R \neq S$ ).

$a^{1}=f^{1}\left(W^{1} p\right)$

$a^{2}=f^{2}\left(W^{2} a^{1}\right)$

Therefore, $a^{2}=f^{2}\left(W^{2} f^{1}\left(W^{1} p\right)\right)$

Where $a^{1}$ represents the hidden layer output, $a^{2}$ represents the output of the output layer, $p$ is the external input to the input layer, $W^{1}$ is the weight connection between input and hidden layer, $W^{2}$ is the weight connection between hidden and output layer, $f^{1}$ is the activation function of the hidden layer and $f^{2}$ is the activation function of the output layer. The outputs of each intermediate layer are the inputs to the following layer. The layers of a multilayer network play different roles. A layer that produces the network output is called an output layer. All other layers are called hidden layers apart from the first layer which is the input layer. The three-layer network shown earlier has one input layer, one output layer and one hidden layer.

\section{Learning Rules}

Learning rule is a procedure for modifying the weights of a network. It may also be referred to as training algorithm, meaning training the network to perform some task. There are three main types of ANN learning rule namely:

1. Supervised Learning

2. Unsupervised Learning

3. Reinforcement Learning

\section{Back Propagation Network}


A back propagation is used when: A large amount of input/output data is available, but you are not sure how to relate it to the output. The problem appears to have overwhelming complexity, but there is clearly a solution. Outputs can be fuzzy, or non-numeric. The term back propagation describes how this type of neural network is trained. Back propagation is a form of supervised training. When using a supervised training method the network must be provided with sample inputs and anticipated outputs [11]. These anticipated outputs will be compared against the actual output from the neural network. Using these anticipated outputs the "back propagation" training algorithm then takes a calculated error and adjusts the weights of the various layers backwards from the output layer all the way back to the input layer. Back propagation can be used with any feed-forward network that uses an activation function which is differentiable [10]. It uses the chain rule in order to compute the derivatives of the squared error with respect to the weights in the hidden layers. It is called back propagation because the derivatives are computed first at the last layer of the network, and then propagated backward through the network.It uses the chain rule in order to compute the derivatives of the squared error with respect to the weights in the hidden layers. It is called back propagation because the derivatives are computed first at the last layer of the network, and then propagated backward through the network.

\section{DESIGN METHODOLOGY}

\section{Development of Neural Network Model}

The developed model of the neural network for effective diagnosis is shown in the block diagram below.

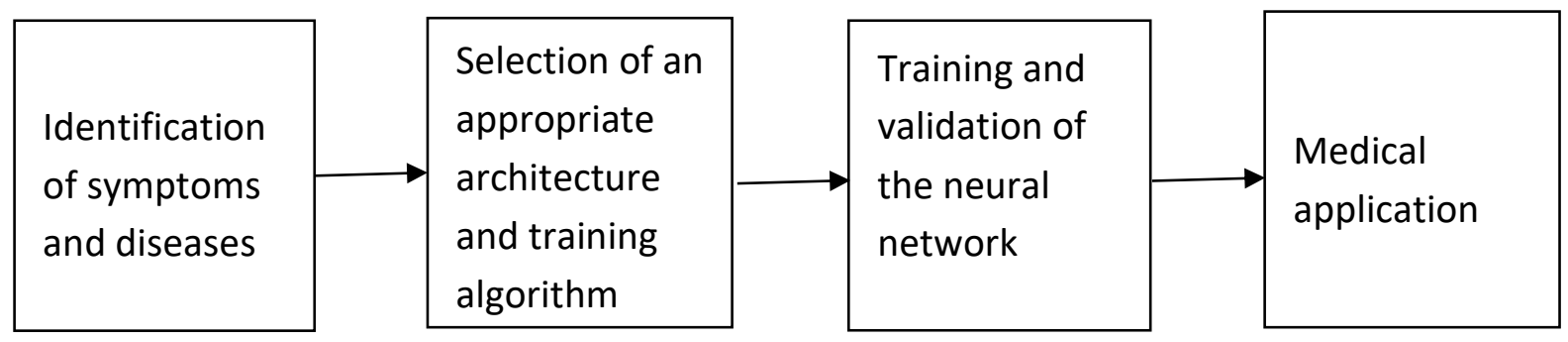

Fig 2: Block diagram for development of the ANN model.

\section{Identification of Symptoms and Diseases}

Symptoms: These are the input parameters required for the neural network to adequately generate and establish a well-known relationship between the symptoms and diseases. They are indications of illness experienced by the patient. These symptoms offer important clues that help doctors to make effective diagnosis. Many at times, the symptoms point to several possible diseases. In the course of this work, thirty-three (33) different inputs were used (see appendix Table 1).

Diseases: These are the output parameters obtained from the ANN network. They are harmful changes that interfere with the normal appearance and structure of the body. Nineteen (19) different diseases were used in this work (see appendix Table 2).

\section{Selection of Neural Network Architecture}

This involves making decisions on:

(i)the number of layers in the network (ii)number of neurons in the input and output layers (iii)number of hidden layers (iv)number of neurons in each of the hidden layers (v) appropriate activation function

One (1) hidden layer is recommended for most practical problem and was used for this work. Table 3 summarizes the difference between the numbers of hidden layers [12] (see appendix Table 3).

In determining the number of neurons in the hidden layer, the following rule of thumb can be used [12].

1. The number of hidden neurons should be in the range between the size of the input layer and the size of the output layer.

2. The number of hidden neurons should be $2 / 3$ of the input layer size, plus the size of the output layer. 
3. The number of hidden neurons should be less than twice the input layer size.

For the purpose of this project, the number of neurons in the hidden layer was chosen to be 10 according to the first law (I).

\section{Activation Function}

The activation function chosen for this work was Log-Sigmoid function. It was chosen because it was differentiable for back propagation. Also it receives any value between plus and minus infinity and returns it between zero and one. A feed forward back propagation neural network was employed for the purpose of this project as shown in fig 3 Back propagation: It describes how the neural network is trained.

Fig 3 A 3-Layers Network

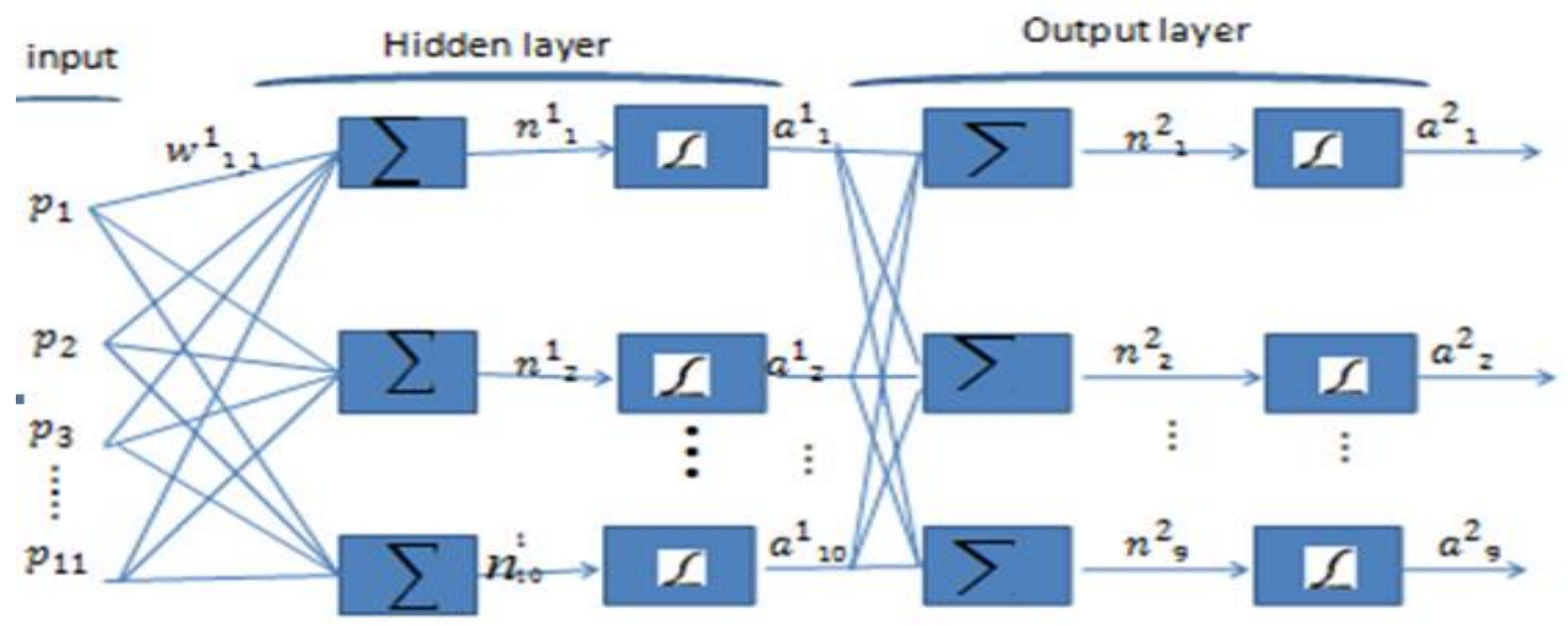

Fig 3: Back propagation

\section{Back Propagation}

Back propagation is a very common method for training multilayer feed forward networks. It was the derivative function that was used during training. The Back propagation training rule is an extended version of the delta rule.

\section{Software Architecture}

The software tool was designed and developed in modules as shown in Fig. 4

Training Module: This module was used for training the neural network. Network parameters were selected such as number of input, hidden and output neurons.

OPERATION MODULE: This is the user interface where one can input various symptoms and patients data like name, sex and age.

OUTPUT MODULE: It gives information on all the possible diseases based on their probability. 


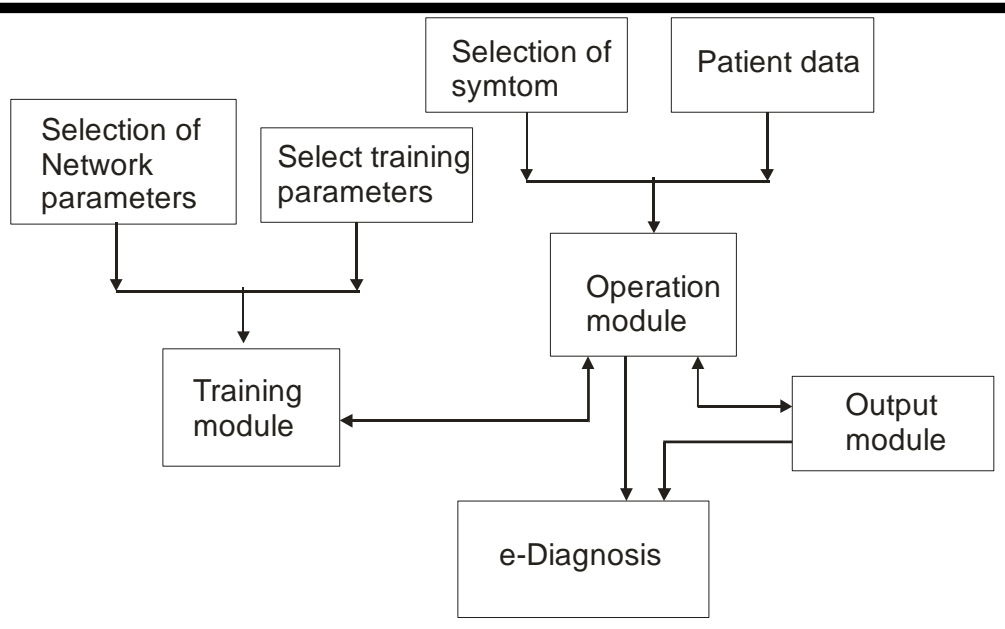

Fig 4: Software design

Algorithm and Flowchart of Training Module

Step 1: Specify number of layers and neurons \& activation function in each Layer

Step 2: Initialize all weights with random values

Step 3: Present a training sample to the neural network

Step 4: Propagate the input forward through the network and obtain an output

Step 5: Compare the network's output to the target from that sample. Calculate the error in each output neuron.

Step 6: If error < a specified value; Back propagate error and adjust weights and GOTO to step 3

Step 7: Stop 


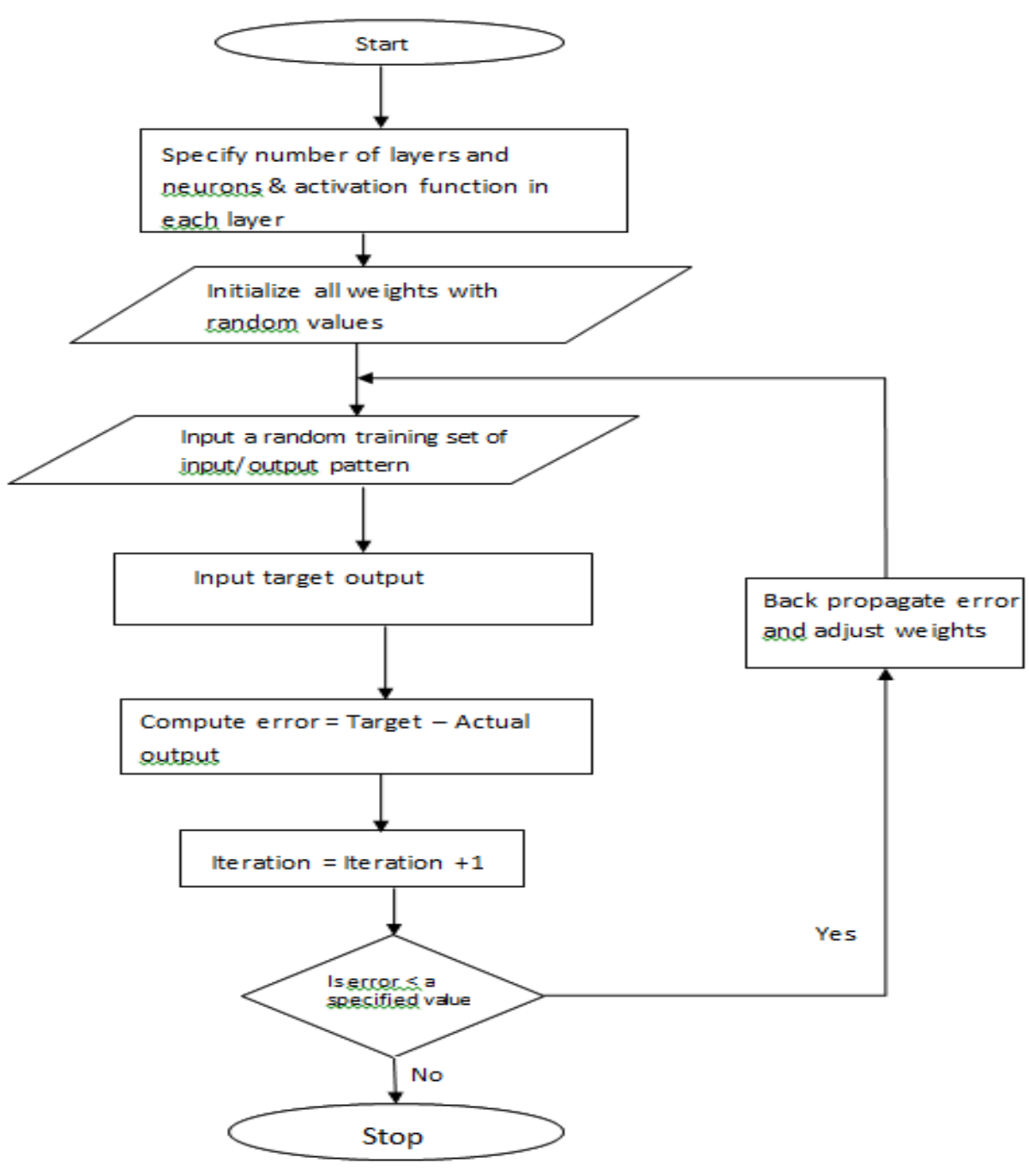

Fig 5: Flowchart for Training Module

\section{Algorithm and Flowchart of Operation Module}

Step 1: Input patients data i.e. name, age and sex

Step 2: Input symptoms

Step 3: Obtain weights saved after training

Step 4: Propagate the input forward and obtain a diagnosis

Step 5: Output the e-diagnosis

Step: 6 Stop 


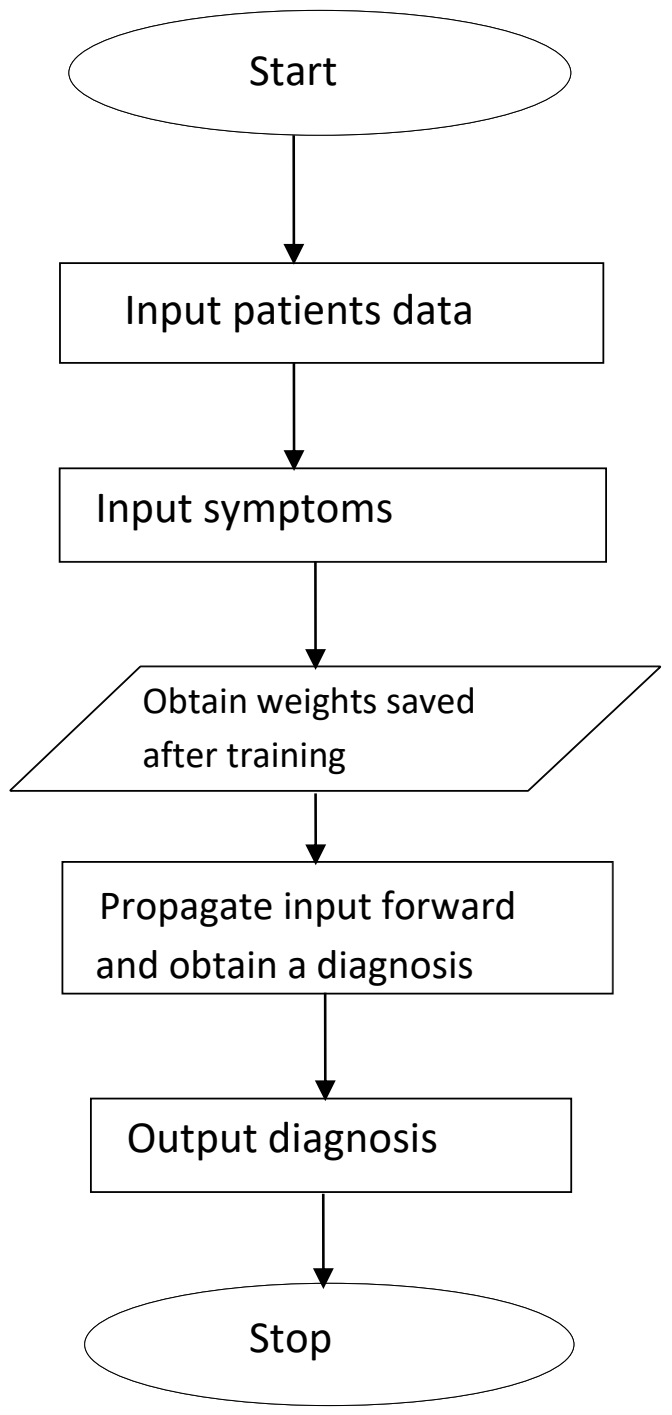

Fig 6: Flowchart for Operation Module

Software implementation: The software tool used for implementation of the algorithm was accomplished using Java programming language. The reason for using this language was because it uses automatic memory allocation, it is object-oriented, it has the ability to run the same program on different systems and its Graphical User Interface (GUI) capabilities. The software was developed in modules as shown in Fig. 7, 8 and 9. Each module has its own GUI that was designed to allow for easy access of the user.

Training module: This module is invoked by clicking on the "Train Network" button of the operation module. It allows the user to train or re-train the neural network. The network and training parameters are first of all selected, weights are obtained by clicking on the "Obtain Weights" button and the network is trained by clicking on "Run". After training, the Root Mean Square Error(RMSE) is obtained. The Reset button enables one to re-train the network. Click on the close button to exit. The training module is shown in Fig 7 
Operation module: This module is where the e-Diagnosis becomes a reality. It allows the user to input personal data (name, age, sex) and various symptoms. As soon as the "Diagnose" button is clicked, the weights obtained after training from the training module is used to process the input through the network thereby giving us a reasonable output. The operation module is shown in Fig 8

Output module: This module enables the user to view the diagnosis (disease(s)). This module is initially hidden and can be invoked by clicking the "Diagnose" button in the operation module. The output module is shown in Fig 9.

Network training: As soon as the network weights are initialized, then the network is ready for training. It is the process of teaching the neural network to be able to represent a particular function [10]. During training, the weights of the network were iteratively adjusted to minimize error. For the purpose of this project, a supervised training algorithm was used. Training a network consist of the actual training and validation of training.

Dataset design: The neural network was taught to diagnose some kind of diseases given a particular set of symptoms as shown in Table 1. To train the network to produce error signal, 675 training data were used. To check whether the network has learned to recognize errors, another 349 validation data were used in other to check for the response of the network to errors on data which were not used in the actual training. Also a program was written in java to generate the training data taking the different combinations of symptoms that gave a particular diagnosis.

The program code in Java was written to simulate the response of the network and perform the learning procedure.

Error analysis: The main aim of training was to reduce the error between the target and actual output. When the error was small it meant that the network learnt very well.

\section{Root Mean Square Error (RMSE)}

It is the RMSE that allows the neural network to know if enough training has taken place. The RMSE is an average error. To take the average error across all training set elements, the size of the training set must be known [9]. The RMSE was calculated by dividing the global error by the product of the training set length and the number of output neurons. The square root of this ratio produced the RMSE.

$\mathrm{RMSE}=[\mathrm{GE} /(\mathrm{L} \times \mathrm{O})] 5$

Where GE is the global error, $\mathrm{L}$ is the length of training set, $\mathrm{O}$ is the number of output neurons

In the course of this work, an experiment was carried out to determine the best training and network parameters to be selected.

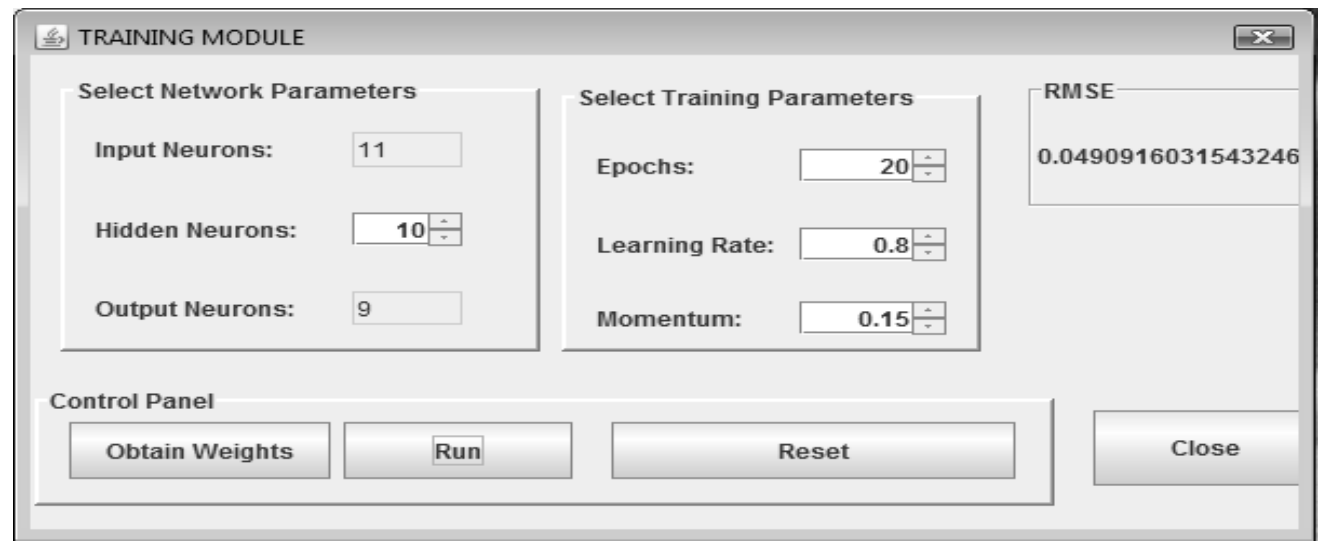

Fig 7: Training module 


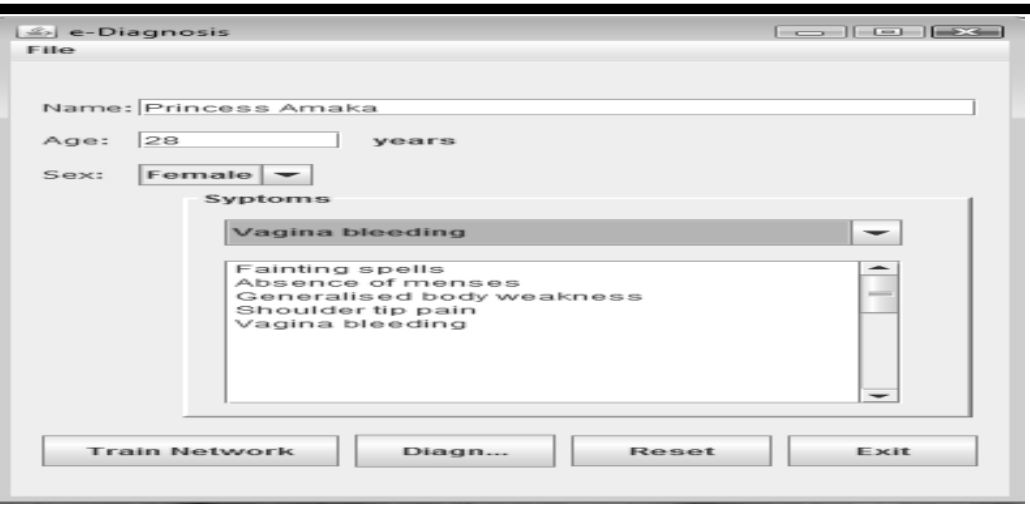

Fig 8: Operation module

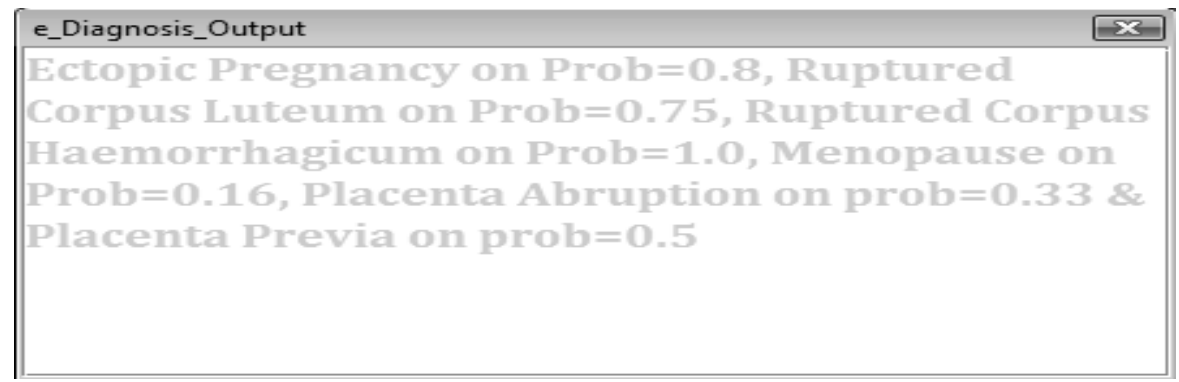

Fig 9: Output module

Validation of Training: Validation of the training process was accomplished by feeding the validation set data to the trained network and the outputs of the network were compared to the target output of the validation set. The training is said to be successful if validation is passed.

\section{RESULTS AND DISSCUSSION}

\subsection{Experimental Result of Training Parameters}

Result of experiment to determine the best combination of training parameters (see appendix Table 4)

From the result shown in the Fig 10, it was discovered that when the RMSE was large, the expected output was far from the actual output but when it was very small, the answer was very close to the target and as a result, an accurate diagnosis was obtained.

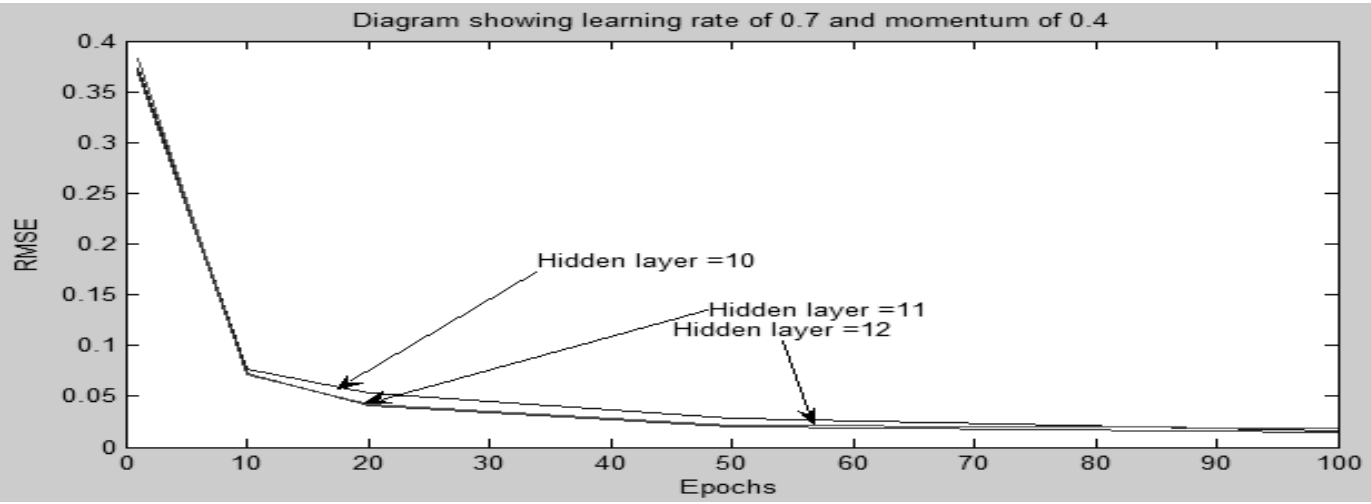


Also, a learning rate $(\alpha)$ of 0.9 and a momentum( $\gamma)$ of .25 with epochs of 50 and 12 neurons in its hidden layer gave the minimal error for the given set of data.

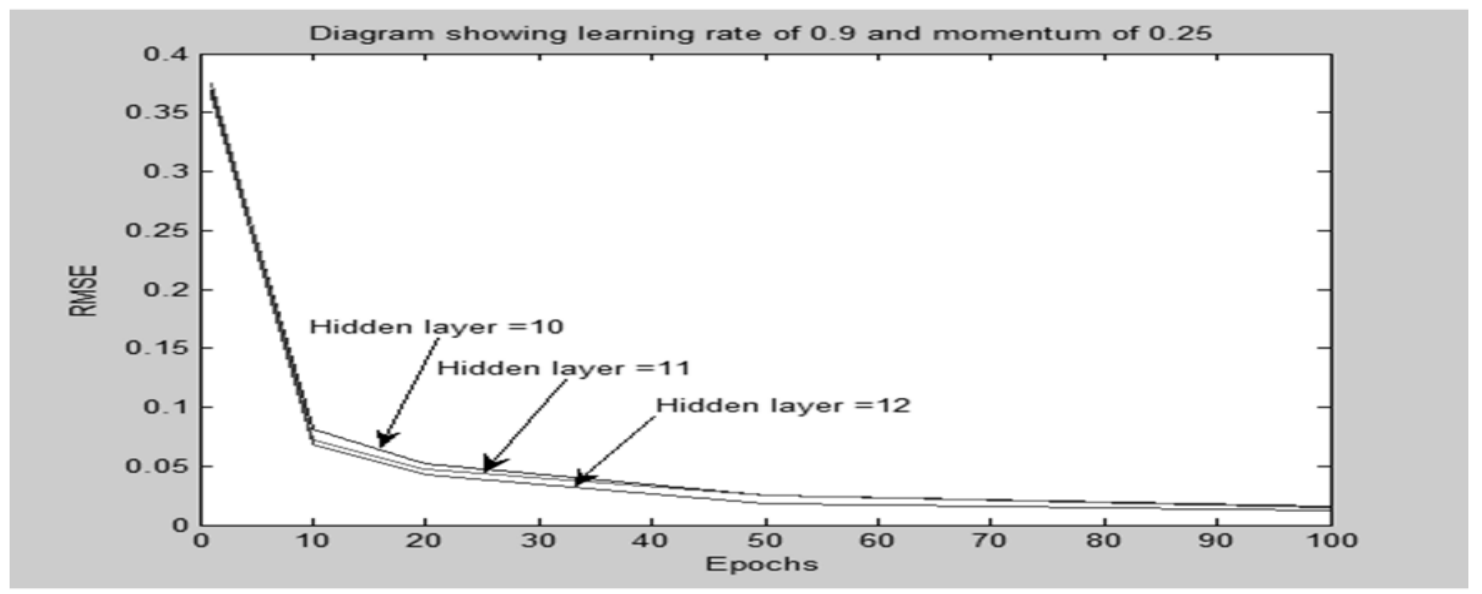

Fig 11: Graph of RMSE versus Epochs for $\boldsymbol{\alpha}=\mathbf{0 . 9}$ and $\boldsymbol{\gamma}=\mathbf{0 . 2 5}$

From the graphs shown in Figures $10 \& 11$, it was observed that the higher the epoch the smaller the RMSE and so the more the network learns. Also, a momentum of 0.25 accelerates convergence faster than that of 0.4 ; however, a learning rate of 0.9 learns faster than that of 0.7

\section{Validation Results}

In the result of training validation, $a=0$ represents the absence of a disease while $a=1$ represents the presence of a disease from the output neurons. The approximation of actual output to nearest whole number gives (0)s and (1)s in determining the absence and presence of disease. Table 5 shows the result of the validation process (see appendix Table 5).

The validation test was passed by all the data meaning that when various inputs were selected, the error obtained (desired minus actual output) was very minimal showing that the training was successful.Also the outputs are nine in number because there were nine (9) neurons at the output layer of the network architecture.

\section{Testing using probability in e-diagnosis}

Probability is one of the main tools used by medical professionals in diagnosis process. It is the likelihood that a disease will occur. In medical diagnosis, there are starting-point likelihoods called "anchor" probabilities. During the subsequent history and examination, the anchor probabilities will undergo a series of upward and downward adjustments according to what the patient has to say (inputs) and what does or does not turn up on her physical examination. The physician individualizes the questions asked and items are examined so that the outcome of each query forces one diagnosis to be more likely and another to be less likely. Thus, diagnosis is a dynamic and sequential process. An illustration was given using Table 4(Test 1). A patient inputted absence of menses which had an anchor probability of $100 \%$ ectopic pregnancy and $16 \%$ menopause occurring (Test 1). Next vaginal itching was(Test 17), then the initial $16 \%(0.16)$ anchor probability of menopause(Test 1 ) got adjusted upward to 0.66 (Test 17) and the initial anchor probability of ectopic pregnancy moved downward from 1.0(Test 1) to 0.2 (Test 18). This, of course, was just a single distinguishing feature, and could not be relied upon to diagnose accurately. The network gathers many such data points to refine the diagnosis. The addition of more symptoms provided another source of facts to distinguish among still-viable possibilities. For the patient above, she also had vagina discharge, painful sexual intercourse/urination and irregular vagina bleeding but had no passage of tissue, shoulder tip pain. These additional symptoms caused (a) the low anchor probability of menopause (0.16) of Test 1 to increase to 0.66 (Test 17), through the symptom of "fainting". (b) Vaginal candidiasis to move upward from 0.8(Test 17) to 1.0(Test 22) through the symptom of "vaginal itching" (c) Gonorrhea to move downward from 1.0(Test 6) to 0.33(Test 17) through the 
symptom of "painful sexual intercourse" (d) Ectopic pregnancy to move downward from 1.0(Test 1) to 0.6(Test 13) through the symptom of "absence of menses". The doctors made their diagnosis and went further to carry out medical test on these patients to confirm their diagnosis. It went along side with testing these patients with the software tool developed and comparing both diagnoses to certify whether the software tool was accurate and efficient. Table 4 shows the results of software diagnosis, doctors' diagnosis and medical test.

Results from software tool, doctor's diagnosis and medical test (see appendix Table 6).

\subsection{Discussion of Result.}

From the results shown in Table 4, it was seen that when different symptoms were selected and the diagnosis button clicked from the software tool, the output gave us diagnosis of all possible diseases that were associated with the symptoms based on what it has learnt. It was observed that the diagnosis was given on probability. This will enable the medical specialist to effectively carry out diagnosis. The result from Tables 4 shows that the software tool was able to identify $82 \%$ of the diseases ( 45 out of 55 cases) as compared with the medical test and $89 \%$ (49 out of 55 cases) as compared with doctor's diagnosis. It was observed that diagnoses with high probability were contained in the doctor's diagnosis. Hence, the software tool will be very useful to medical doctors in the field of obstetrics and gynecology to enhance their diagnosis. Also, it was shown that diagnosis with a probability of one was certain that the disease was present. While those with probability less than one was not too certain but might be present. Also when 'select' was inputted, the diagnosis was 'no illness' indicating that all eleven neurons at the input layer of the network were zero. In addition, when a particular symptom was inputted more than once, the diagnosis was 'go and see a medical practitioner'. It is apparent that before operations can be effected the network has to be trained and the correct set of weights that best represents the relationship between the input and the output parameters must be established.

\section{Disadvantages}

Set of weights was obtained by severally adjusting the learning rate and momentum, varying the neurons in the hidden layer and increasing the number of epochs. It was observed that e-Diagnosis would be very inaccurate with the wrong set of weights. The software tool was unable to diagnose six (6) different diseases because they were not included in the data collected.

\section{CONCLUSION}

Current medical service systems are lacking in terms of offering accurate diagnosis, and early detection of disease symptoms and problems in patient's health state. It's not too much of a stretch of the imagination to realize that telemedicine will soon be just another way to see a health specialist, just as using any other software on your system. Also, it has been theorized that medical doctors could have an 'e-Diagnosis System' as part of their home entertainment centers.

The importance of an appropriate design methodology in this application was demonstrated. The neural network is an intelligent programming technique capable of solving medical diagnosis problem, it also provided accurate results. In fact, it successfully represents the function it was trained to perform. While acknowledging the limitations of the software tool, this study also represents a positive step towards developing an exhaustive tool for telemedicine applications in the real world context. In conclusion, medical diagnosis provided by artificial intelligence applications can save lives, reduce cost and be useful to medical doctors.

\section{RECOMMENDATIONS}

The developed software is capable of carrying out diagnosis in obstetrics and gynaecology only due to the scope of the work. Therefore it is recommended that the scope should be extended to other areas of medicine such as pediatrics, internal medicine, radiology etc. The software application could be used by doctors to double-check their diagnosis. This provides a very less tedious means of carrying out diagnosis with reduction in diagnosis errors and is relatively much faster. It could also be used in cases of emergency where the identification of a disease(s) is very necessary 
before seeing a specialist so that treatment can commence. In other words, patients can be checked for diseases quickly and painlessly and thus detecting diseases at early stages.

\section{REFERENCES}

[1] Norris, A. (2002). Essentials of telemedicine and telecare. John Wiley \& Sons: Chichester

[2] Dalton, J. J. (1995). Artificial Neural Networks for Decision Support in Clinical Medicine. Annals of Medicine, Informa Healthcare Plc. Vol. 27, No. 5, Pages 509-517.

[3] Nicks, V. (2009). AI Applications in online medical diagnosis: using a neural network data mining solution to diagnose illness. Journal of Investigate Dermatology. Cattedra di Endocrinolgia University, Parma, Italy. Vol 122, pp. 443-449.

[4] Perednia, D. A. (1995). Telemedicine technology and clinical applications. JAMA 273, 66 .American Medical Association, Chicago, pp. 483-488.

[5] Georgios, G.M.D. (2008). ECG Signal Recording, Processing and Transmission using a mobile phone. PETRA; Vol. 282Proceedings of the 1st international conference on Pervasive Technologies Related to Assistive Environments. ACM, New York, NY, USA.

[6] Bashur, R. \&Kathan, C. (1999). Human factors in telemedicine. Journal of Telemedicine and e-Health, Vol 5:127128.

[7] Huston, T.L. (2000). Is Telemedicine a practical reality? IEEECOMMUNICATIONS OF THE ACM Vol. 43, No. 6, 91-95.

[8] Epidemiol, J. C. (1996). Advantages and disadvantages of using artificial neural networks versus logistic regression for predicting medical outcomes. Institute for Clinical Evaluative Sciences, North York, Ontario, Canada. , 49(11):1225-31.

[9] Daniel, G. (2007). Principles of Artificial Neural Network, University of Illinois, Chicago, USA. $2^{\text {nd }}$ Edition. Advanced Series on Circuits and Systems - Vol.6. Singapore: World Scientific Publishing Co. Pte. Ltd.

[10] Martin, T. \& Howard, B. (2002). Neural Network Design. PWS Publishing Company, Thomson Learning, USA. [11] McCollum, P. (2008). An Introduction to Back-Propagation Neural Networks. Encoder: The Newsletter of the saetle Robotics Society, Vol 25, pp. 23-25.

[12] Heaton, J. (2005). Programming Neural Networks in Java, McGraw Hill Publications, New York.

[13] Jigneshkumer L. Pattel, Ramash k. Goyal (2007). Application of Artificial Neural Network in Medical Science Current Clinical Pharmacology Vol. 2, Issue 3.

[14] DancoMitaah M. Pattis, Edwin D.C., Subido and Milo T. Bugtar (2018) https://dol.org/10.1063/5023979.

[15] QuetharaKadhim, Alshayea (2011). Artificial Nueral Network in Medical Diagnosis. DCSS Intrnational Journal of Computer Science, Issues, Vol 8, Issue 2. March 2011

[16] Nida Shahid, Tim Rapon and Whitney Rappon (2019). Application of Artificiall Neural Network in Healthcare Organisational Decision Making. 